\title{
Surgery in Masaoka stage IV thymic carcinoma: a propensity-matched study based on the SEER database
}

\author{
Cong Ye ${ }^{1 \#}$, Minwei Bao ${ }^{1 \#}$, Huiting Li $^{2}$, Xiaogang Liu ${ }^{1}$, Gening Jiang ${ }^{1}$, Weixi Wang ${ }^{3}$ \\ ${ }^{1}$ Department of Thoracic Surgery, ${ }^{2}$ Department of Respiratory, Shanghai Pulmonary Hospital, Tongji University School of Medicine, Shanghai \\ 200433, China; ${ }^{3}$ Department of Geriatrics, Zhongshan Hospital, Fudan University, Shanghai 200032, China \\ Contributions: (I) Conception and design: W Wang, C Ye; (II) Administrative support: H Li; (III) Provision of study materials or patients: X Liu, W \\ Wang, C Ye; (IV) Collection and assembly of data: M Bao, C Ye; (V) Data analysis and interpretation: G Jiang, W Wang; (VI) Manuscript writing: \\ All authors; (VII) Final approval of manuscript: All authors. \\ \#These authors contributed equally to this work. \\ Correspondence to: Dr. Weixi Wang, MD. Department of Geriatrics, Zhongshan Hospital, Fudan University, No. 180 Fenglin Road, Xuhui District, \\ Shanghai 200032, China. Email: wangweixi163@163.com.
}

Background: Thymic carcinoma is a type of rare and highly malignant tumor. Limited information was available on prognostic factors of late-stage thymic carcinoma. The aim of this study was to identify factors that impact prognosis and to define the relationship between survival and surgical intervention in patients with Masaoka stage IV thymic carcinoma.

Methods: From 1973 to 2015, a total of 311 consecutive patients were enrolled in this study with pathologic confirmed Masaoka stage IV thymic carcinoma from the Surveillance, Epidemiology, and End Results database. Kaplan-Meier analyses, Cox-regression analyses and propensity score matching (PSM) were performed to evaluate prognosis.

Results: In the multivariate analysis, larger tumor size, distant metastasis and positive lymph node status were associated with poorer outcome. After PSM, no receipt of surgery was the prognostic factor indicating poorer survival [hazard ratio (HR) 1.985, 95\% confidence interval (CI) 1.007-3.913, $\mathrm{P}=0.048$ for overall survival (OS); HR 1.649, 95\% CI: 1.009-2.697, P=0.046 for disease-specific survival (DSS)]. Subgroup analysis indicated that significantly improved survival with surgery was observed in patients who were $<60$ years (HR 0.48, 95\% CI: 0.32-0.72), female (HR 0.37, 95\% CI: 0.23-0.60), Caucasian (HR 0.56, 95\% CI: 0.40-0.77), with larger tumor size ( $\geq 7.0 \mathrm{~cm}$, HR 0.42, 95\% CI: 0.25-0.69), with (HR 0.60, 95\% CI: 0.39-0.90) or without distant metastasis (HR 0.46, 95\% CI: 0.26-0.83), and node-positive (HR 0.56, 95\% CI: $0.38-0.82)$.

Conclusions: Surgical treatment could be beneficial in patients with Masaoka stage IV thymic carcinoma. This SEER based analysis revealed the role of surgical resection and the favorable effect of surgery in specific thymic carcinoma subgroups.

Keywords: Thymic carcinoma; SEER program; propensity score matching (PSM); surgery

Submitted Sep 23, 2019. Accepted for publication Dec 13, 2019.

doi: $10.21037 /$ jtd.2019.12.111

View this article at: http://dx.doi.org/10.21037/jtd.2019.12.111

\section{Introduction}

Thymic carcinoma is a type of rare and highly malignant tumor originating from the thymic epithelium, associated with malignant cytological features (high potential for metastasis and aggressive tumor invasiveness) (1). The World Health Organization (WHO) histologic classification classified thymic carcinoma as a separate entity (2), defining it different from thymic neuroendocrine tumors and thymomas (3). Most patients with thymic carcinoma have 
various clinical symptoms at the time of diagnosis. Primary tumors extended to the adjacent mediastinum and with positive lymph node (LN) status accounted for nearly $80 \%$ and $40 \%$ of thymic carcinoma patients, respectively $(4,5)$. The 5-year survival rate of thymic carcinoma was reported from $28 \%$ to $67 \%$ in literatures $(6,7)$. Thymoma is of an extremely rare prevalence $(0.17 / 100,000)$, yet thymic carcinoma is even less common than thymoma, accounting for approximately $15-20 \%$ of all thymic epithelial tumors $(7,8)$. Administration of surgery is recommended as the gold standard treatment for resectable thymic carcinoma, with adjuvant therapies such as chemotherapy and radiation therapy (9). However, for advanced stage thymic carcinomas, most of which are unresectable, the prognostic impact of surgical intervention remains unknown. Because of its rarity, most existing literatures on thymic carcinoma are small-sample, single-center and retrospective studies, and researches focusing on late-stage thymic carcinoma are even less. Multidisciplinary cooperation, combining with preoperative chemotherapy can be considered as an option in unresectable diseases (10), while it has not been reached as a consensus owing to the low incidence of thymic carcinomas (11).

To address this knowledge gap, we quired the Surveillance, Epidemiology and End Results (SEER) database to establish a retrospective study, in order to identify factors that exert impact on the prognosis of patients with Masaoka stage IV thymic carcinoma, furthermore, to better interpret the relationship between surgery intervention and survival outcome in patients with late-stage thymic carcinoma. In general, the present research can shed light on the prognostic implications of surgical resection in the contemporary era.

\section{Methods}

\section{Data collection}

The publicly available SEER database covered approximately $30 \%$ of the American population (12). SEER*Stat software (version 8.3.5; National Institutes of Health, Bethesda, MD) was used to extract clinicopathologic and survival information from 1973 to 2015 . The primary site variable was defined as tumors of thymus, and histology type of thymic carcinoma was identified in accordance with the International Classification of Diseases (ICD) codes: $8020,8023,8033,8070,8082,8123,8140,8260,8310$, $8430,8480,8560,8576$, and 8586 , all accompanied with the malignant behavior code (/3) (13). The available inclusion criteria included: (I) age $\geq 18$ years; and (II) Masaoka stage IV thymic carcinoma. Patients were excluded if: (I) unavailable information of surgery or extent of surgery; (II) not the first primary tumor or with other malignancies; and (III) survival time less than one month (to rule out immediate post-operative mortality). Surgery modalities were classified into total/radical resection and non-total resection (including local tumor excision, partial removal, and debulking surgery). Pertinent information of primary tumor extension, LN status, and metastasis status were extracted from the SEER registry for staging. Masaoka stage I-IIA, IIB, III, and IV were identified as localized or organconfined, extending to adjacent connective tissue, extending to adjacent organs or structures in the mediastinum, and further contiguous extension, respectively. Patients with positive $\mathrm{LN}$ status or with distant metastasis were classified as Masaoka stage IV (14). The present study is based on a publicly available database, thus, it was exempted from institutional review board approval.

\section{Propensity score matching (PSM)}

Selection bias is always a concern especially in a retrospective study. A propensity score is the conditional probability of assignment to a specific intervention given a vector of baseline covariates (15), which is often applied to reduce selection bias and balance the distributions of confounding factors (16). In the present study, we conducted a non-parsimonious logistic regression model to calculate the propensity score regarding clinicopathologic characteristics across the surgery group and the nonsurgery group. Parameters significant in the univariate logistic regression models were entered into a multivariate logistic regression model. PSM was performed using 1:1 nearest neighbor matching with a caliper of 0.02 to accept a matched pair (17). The variables were verified as balanced in the matched population for further investigation.

\section{Statistical analysis}

Kaplan-Meier analysis was conducted to calculate the survival rate and Cox proportional hazards model was conducted to adjust variables with $\mathrm{P}<0.1$ in the univariate analyses. Statistically significant result was obtained if twosided $\mathrm{P}<0.05$. All analyses were performed with SPSS Statistics 25.0 (IBM, Armonk, NY, USA). The primary endpoint of the current research was overall survival (OS) 


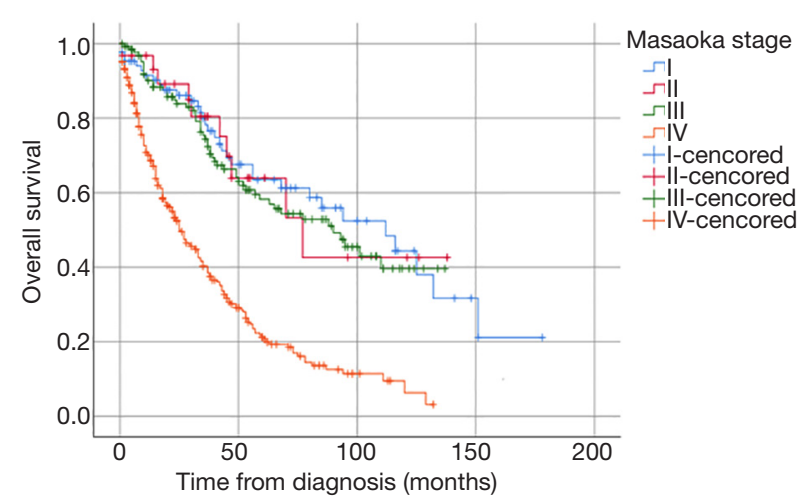

Figure 1 Overall survival analysis of patients with thymic carcinoma in different Masaoka stages.

and disease-specific survival (DSS), defined as the interval between the diagnosis and death from any cause and the time from the diagnosis of cancer to death of thymic carcinoma respectively.

\section{Results}

From 1973 to 2015, a total of 660 thymic carcinoma cases were included according to the eligibility criteria. The number of patients classified into stage I-IIA, IIB, III, and IV were $86,31,129$, and 311 , with the 5 -year OS of $63.4 \%$, $63.9 \%, 58.2 \%$ and $21.2 \%$, respectively. Survival analysis revealed that stage I-IIA, IIB and III patients resembled in OS, but the stage IV patients had a significantly poorer survival than the other three stages (Figure 1). Thus, stage IV patients had predominance in population as well as the worst prognosis.

\section{Baseline characteristics}

A total of 311 records with thymic carcinoma of Masaoka stage IV were identified. Clinicopathological characteristics including age, gender, ethnicity, grade, LN status, tumor size, distant metastasis, type of surgery, and multimodality treatment were collected in Table 1. Male (61.1\%) and the Caucasian $(71.1 \%)$ were predominant in the population with the median age of 60 years (range, 20-92 years). The median size for thymic carcinoma was $7.0 \mathrm{~cm}$ (range, $1.1-17.0 \mathrm{~cm}$ ). Positive LN status was found in $55.3 \%$ patients and distant metastasis was found in $58.5 \%$ patients. The top four metastasis sites are lung, bone, liver and brain, sorted by incidence from high to low. Approximately $40 \%$ patients underwent surgery, while radiation therapy was prescribed in $31.2 \%$ patients. About $75.9 \%$ patients undertook chemotherapy, whereas the SEER database didn't give any information about the order between surgery and chemotherapy and didn't distinguish between no receipt of chemotherapy and unknown information of chemotherapy.

\section{Prognostic factors}

Univariate Kaplan-Meier analysis of Masaoka stage IV patients was recorded in Table 2. Significantly degradations of survival was observed in patients with no receipt of radiation treatment $(\mathrm{P}<0.001$ for both OS and DSS), no receipt of surgery $(\mathrm{P}<0.001$ for both OS and DSS), larger tumor size $(\geq 7.0 \mathrm{~cm}, \mathrm{P}=0.004$ for $\mathrm{OS}$ and $\mathrm{P}=0.003$ for DSS), positive nodal status ( $\mathrm{P}=0.044$ for $\mathrm{OS}$ and $\mathrm{P}=0.070$ for DSS) and distant metastasis $(\mathrm{P}<0.001$ for both OS and DSS, especially metastasis at brain or liver). When further exploring in surgery modalities, total/radical resection showed superior DSS rates [non-total resection vs. total/ radical resection: hazard ratio (HR) 1.821, 95\% confidence interval (CI): 1.094-3.033, $\mathrm{P}<0.001$; no surgery vs. total/ radical resection: HR 2.483, 95\% CI: $1.607-3.836, \mathrm{P}<0.001$; non-total resection $v s$. no surgery: HR $0.733,95 \% \mathrm{CI}$ : $0.507-1.062, \mathrm{P}=0.101]$. Total/radical resection resulted in superior OS rates than did no surgery (no surgery $v$ s. total/radical resection: HR 2.098, 95\% CI: 1.449-3.036, $\mathrm{P}<0.001$ ), while there was no significant difference between total/radical resection and non-total resection $(\mathrm{P}=0.053)$ nor between no receipt of surgery and non-total resection $(\mathrm{P}=0.084)$ in OS.

Table 3 demonstrated the result of multivariate analysis. Larger tumor size had an intimate relationship with poorer OS (HR 1.813, 95\% CI: 1.193-2.754; $\mathrm{P}=0.005)$ and DSS (HR 1.968, 95\% CI: 1.243-3.115; P=0.004), while negative LN status and no distant metastasis was associated with better OS (HR 0.570, 95\% CI: 0.349-0.931; P=0.025 and HR 0.563, 95\% CI: 0.333-0.958; $\mathrm{P}=0.034$, respectively). However, no significant difference was found in both OS and DSS in patients receiving radiation therapy or different surgical modalities.

A stratified exploratory analysis was performed to assess the appropriate treatment for patients with Masaoka stage IV thymic carcinoma (Table 4 and Figure S1). Significant reductions in OS and DSS were observed in patients receiving non-total resection without radiotherapy, while no significant difference was discovered for other subgroups. However, Patients receiving total/radical resection with radiotherapy tended to yield superior survival rate with the 
Table 1 Baseline characteristics of thymic carcinoma at Masaoka stage IV ( $\mathrm{n}=311)$

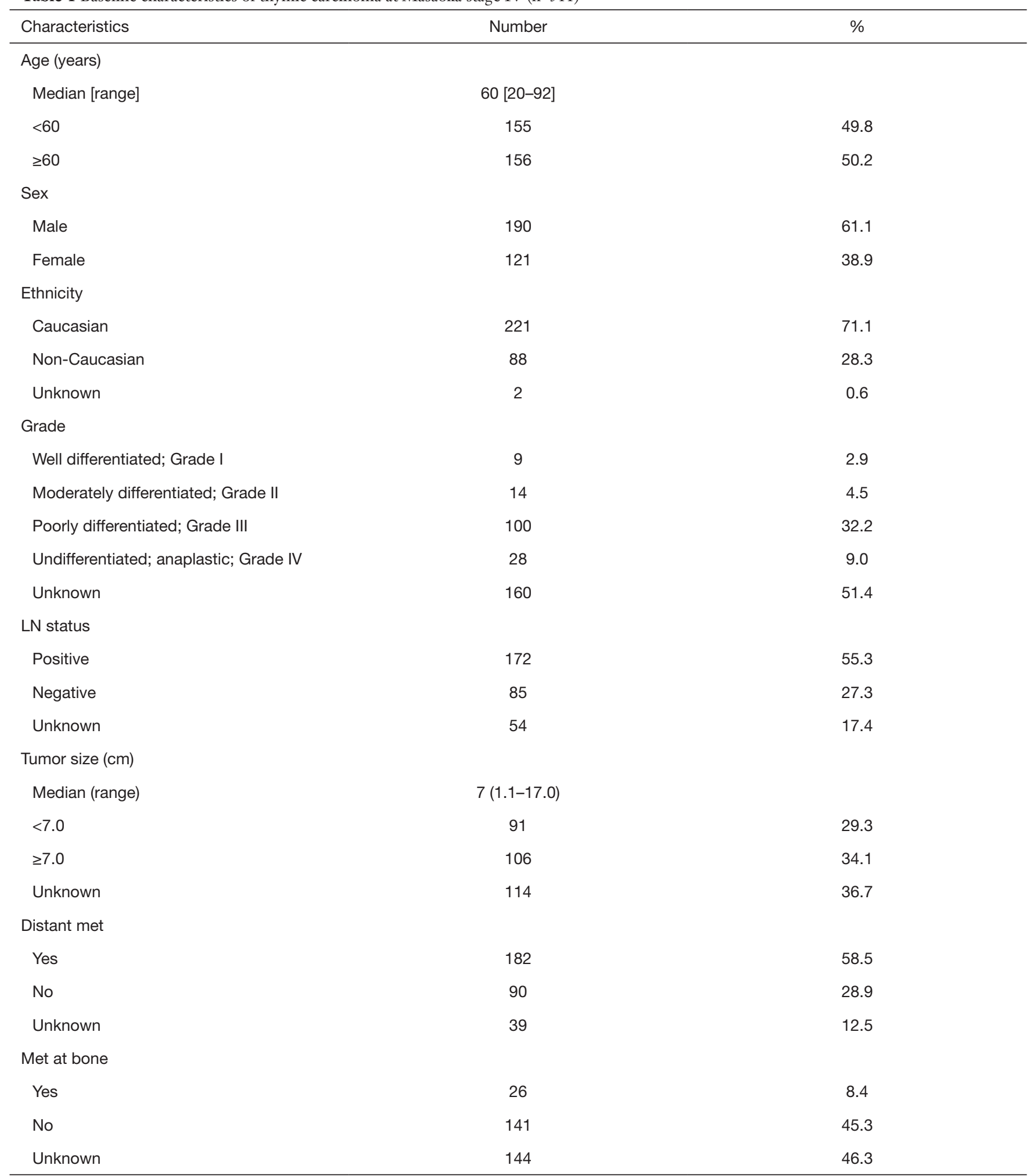

Table 1 (continued) 
Table 1 (continued)

\begin{tabular}{|c|c|c|}
\hline Characteristics & Number & $\%$ \\
\hline \multicolumn{3}{|l|}{ Met at brain } \\
\hline Yes & 7 & 2.3 \\
\hline No & 159 & 51.1 \\
\hline Unknown & 145 & 46.6 \\
\hline Yes & 26 & 8.4 \\
\hline No & 141 & 45.3 \\
\hline Unknown & 144 & 46.3 \\
\hline \multicolumn{3}{|l|}{ Met at lung } \\
\hline Unknown & 145 & 46.6 \\
\hline \multicolumn{3}{|l|}{ Surgery } \\
\hline Yes & 124 & 39.9 \\
\hline No & 187 & 60.1 \\
\hline \multicolumn{3}{|l|}{ Extent of surgery } \\
\hline Total/radical resection & 64 & 20.6 \\
\hline Non-total resection & 60 & 19.3 \\
\hline No surgery & 187 & 60.1 \\
\hline No/unknown & 75 & 24.1 \\
\hline
\end{tabular}

LN, lymph node; met, metastasis.

5 -year OS rates of $37.6 \%$ and 5-year DSS rates of $42.3 \%$. Due to the limitation of sample size, these differences are not statistically significant.

\section{Survival analysis and prognostic factors after PSM}

To minimize selection bias, a PSM analysis of the administration of surgery was performed and the KaplanMeier survival curves of matched and unmatched dataset were illustrated in Figure 2. The 5-year OS and DSS of surgery group $v s$. non-surgery groups were $28.4 \% v s$.
$16.0 \%$ and $31.1 \%$ vs. $15.4 \%(\mathrm{P}<0.001)$ respectively in the unmatched population. Table 5 listed the balances of each factor in the unmatched and matched population. In the matched population, there were no significant factors associated with the receipt of surgery. The categorized variables, such as age ( $\geq 60$ and $<60$ years), sex (male and female), race (Caucasian and non-Caucasian), radiation treatment (yes and no), tumor size $(\geq 7.0$ and $<7.0 \mathrm{~cm})$, distant metastasis (yes and no) and LN metastasis (yes and no) showed the well-balanced result. In the matched dataset $(\mathrm{n}=124)$, the 5 -year OS and DSS rate of the surgery group 
Table 2 Univariate Kaplan-Meier analysis of OS and DSS

\begin{tabular}{|c|c|c|c|c|c|c|}
\hline Characteristics & \multicolumn{3}{|c|}{ OS } & \multicolumn{3}{|c|}{ DSS } \\
\hline \multicolumn{7}{|l|}{ Age (years) } \\
\hline$<60$ & 39.7 & 18.6 & 0.606 & 39.7 & 20.6 & 0.643 \\
\hline$\geq 60$ & 41.0 & 24.3 & & 41.1 & 23.0 & \\
\hline Sex & & & 0.552 & & & 0.574 \\
\hline Female & 42.5 & 26.6 & & 42.3 & 27.6 & \\
\hline Ethnicity & & & 0.112 & & & 0.187 \\
\hline Caucasian & 45.7 & 23.6 & & 45.4 & 23.3 & \\
\hline Non-Caucasian & 28.0 & 15.5 & & 27.5 & 18.0 & \\
\hline Surgery & & & $<0.001$ & & & $<0.001$ \\
\hline Yes & 51.7 & 28.4 & & 52.9 & 31.1 & \\
\hline No & 32.2 & 16.0 & & 32.2 & 15.4 & \\
\hline Extent of surgery & & & $<0.001$ & & & $<0.001$ \\
\hline Total/radical resection & 63.7 & 35.3 & & 70.0 & 41.1 & \\
\hline Non-total resection & 39.9 & 21.6 & & 38.3 & 22.3 & \\
\hline No surgery & 32.2 & 16.0 & & 32.2 & 15.4 & \\
\hline Tumor size $(\mathrm{cm})$ & & & 0.004 & & & 0.003 \\
\hline$<7.0$ & 58.1 & 31.8 & & 60.3 & 36.5 & \\
\hline Positive & 36.5 & 20.0 & & 38.1 & 20.4 & \\
\hline Negative & 52.2 & 31.1 & & 49.7 & 33.5 & \\
\hline Met at bone & & & 0.194 & & & 0.168 \\
\hline Yes & 42.8 & 21.4 & & 42.1 & 42.1 & \\
\hline No & 41.1 & 23.6 & & 43.7 & 26.4 & \\
\hline Met at brain & & & 0.001 & & & 0.001 \\
\hline Yes & 0.0 & 0.0 & & 0 & 0.0 & \\
\hline No & 41.5 & 23.9 & & 43.2 & 27.2 & \\
\hline Met at liver & & & 0.001 & & & 0.001 \\
\hline Yes & 9.1 & 0.0 & & 11.2 & 0.0 & \\
\hline No & 43.1 & 26.7 & & 44.7 & 30.3 & \\
\hline Met at lung & & & 0.339 & & & 0.234 \\
\hline Yes & 42.9 & 8.6 & & 40.3 & 13.4 & \\
\hline No & 39.8 & 28.3 & & 43.9 & 31.2 & \\
\hline
\end{tabular}

LN, lymph node; OS, overall survival; DSS, disease-specific survival; met, metastasis. 
Table 3 Cox-regression analysis for prognostic factors

\begin{tabular}{|c|c|c|c|c|}
\hline Variables & \multicolumn{2}{|c|}{ OS } & \multicolumn{2}{|c|}{ DSS } \\
\hline LN status & & 0.025 & & 0.066 \\
\hline Yes & 1 & & 1 & \\
\hline No & $0.570(0.349-0.931)$ & & $0.602(0.351-1.033)$ & \\
\hline$<7.0$ & 1 & & 1 & \\
\hline$\geq 7.0$ & $1.813(1.193-2.754)$ & & $1.968(1.243-3.115)$ & \\
\hline Distant metastasis & & 0.034 & & 0.059 \\
\hline Yes & 1 & & 1 & \\
\hline Non-total resection & $1.245(0.680-2.280)$ & 0.478 & $1.561(0.788-3.093)$ & 0.202 \\
\hline No surgery & $1.662(0.885-3.120)$ & 0.114 & $1.784(0.855-3.722)$ & 0.123 \\
\hline Radiation treatment & & 0.4 & & 0.333 \\
\hline Yes & 1 & & 1 & \\
\hline No & $1.256(0.739-2.133)$ & & $1.356(0.732-2.511)$ & \\
\hline
\end{tabular}

$\mathrm{Cl}$, confidence interval; LN, lymph node; OS, overall survival; DSS, disease-specific survival; met, metastasis.

Table 4 Subgroup analysis of OS and DSS according to surgery procedure and radiotherapy

\begin{tabular}{|c|c|c|c|c|c|c|c|c|}
\hline Subgroup & \multicolumn{4}{|c|}{ OS } & \multicolumn{4}{|c|}{ DSS } \\
\hline $\begin{array}{l}\text { Non-total resection with } \\
\text { radiotherapy }(n=34)\end{array}$ & 42.7 & 21.6 & $1.532(0.865-2.715)$ & 0.144 & 42 & 23.6 & $1.614(0.865-3.012)$ & 0.132 \\
\hline $\begin{array}{l}\text { Non-total resection } \\
\text { without radiotherapy } \\
(n=26)\end{array}$ & 37 & 23.1 & $1.997(1.115-3.576)$ & 0.020 & 34 & 22.9 & $2.221(1.149-4.291)$ & 0.018 \\
\hline
\end{tabular}

$\mathrm{Cl}$, confidence interval; OS, overall survival; DSS, disease-specific survival; HR, hazard ratio.

were both higher in that of the non-surgery group $(32.7 \%$ vs. $14.9 \%, \mathrm{P}=0.027$ for $\mathrm{OS} ; 38.4 \%$ vs. $13.1 \%, \mathrm{P}=0.010$ for DSS).

The results of univariate Kaplan-Meier analysis after
PSM are shown in Table 6. Significantly degradations of survival were observed in patients with male sex $(\mathrm{P}=0.009$ for OS and $\mathrm{P}=0.008$ for DSS), no receipt of radiation therapy ( $\mathrm{P}=0.029$ for $\mathrm{OS}$ and $\mathrm{P}=0.028$ for DSS) or without 
A

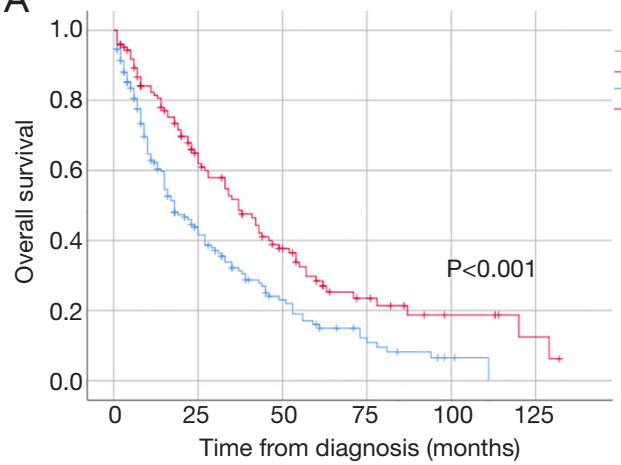

C

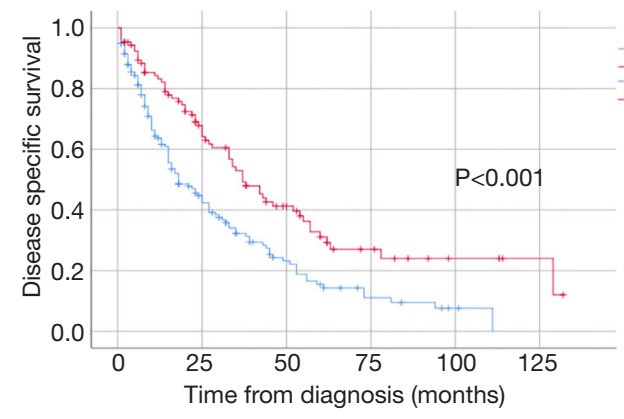

B

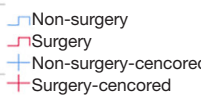

+ Non-surgery-cencor
+Surgery-cencored

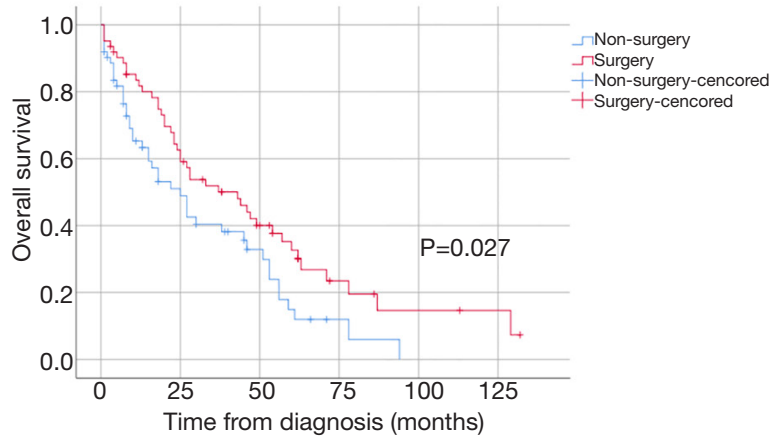

D

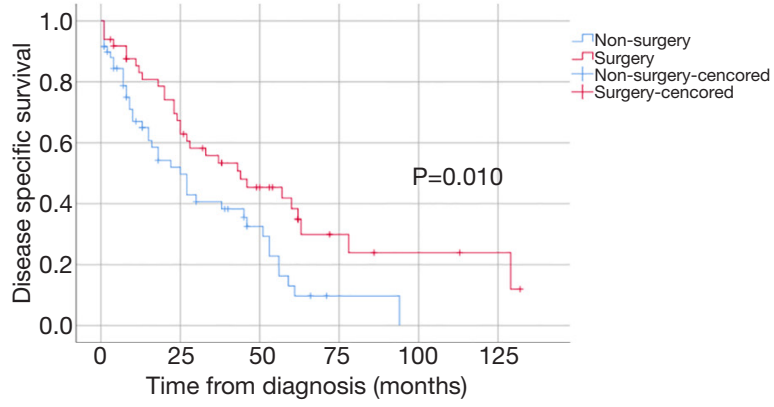

Figure 2 Overall survival of patients with and without surgery, before (A) and after (B) propensity score matching (PSM). Disease-specific survival of patients with and without surgery, before (C) and after (D) PSM.

surgery ( $\mathrm{P}=0.027$ for $\mathrm{OS}$ and $\mathrm{P}=0.010$ for DSS). Table 7 shows the result of Cox-regression analysis. Among the variables, no receipt of surgery was the only prognostic factor indicating poorer survival in OS and DSS (HR 1.985, 95\% CI: $1.007-3.913, \mathrm{P}=0.048$ for OS and HR $1.649,95 \%$ CI: $1.009-2.697, \mathrm{P}=0.046$ for DSS).

\section{Subgroup analysis}

To determine the patients who had the benefit from surgery intervention, we conducted an exploratory subgroup analysis (Figure 3). Significantly superior survival of surgery was observed in patients with age of diagnosis $<60$ years (HR: 0.48, 95\% CI: 0.32-0.72), female sex (HR: 0.37, 95\% CI: 0.23-0.60), Caucasian ethnicity (HR: 0.56, 95\% CI: $0.40-0.77)$, larger tumor size ( $\geq 7.0 \mathrm{~cm}, \mathrm{HR}: 0.42,95 \%$ CI: 0.25-0.69), positive LN statues (HR: $0.56,95 \%$ CI: 0.38 0.82 ), and with (HR: $0.60,95 \%$ CI: $0.39-0.90$ ) or without distant metastasis (HR: 0.46, 95\% CI: 0.26-0.83).

\section{Discussion}

Thymic carcinoma is a type of rare disease with aggressive nature, leading to high morbidity and mortality. Survival rates varied according to stage (stages I-II: 91\%; stages III-IV: $31 \%$ ) and resectability (including completeness of resection) (18). The Masaoka staging system is widely used for thymic carcinomas and advanced Masaoka stage was associated with poor survival (19-21). Similar results were also observed in the present study, patients with Masaoka stage IV thymic carcinoma accounted for nearly half of the population with the 3-, 5- and 10-year OS of $40.3 \%, 21.2 \%$, and $6.3 \%$, respectively, reaching the worst survival among other stages from the same SEER database $(\mathrm{P}<0.001)$. It is reported that administration of surgery is the preferred modality of treatment for thymic carcinoma (9), however, the role of surgery approach has been undefined in stage IV thymic carcinoma. Thus, we specifically analyzed the survival of patients with Masaoka stage IV thymic carcinoma to evaluate the role of surgery in outcome. Surgery treatment had an intimate relationship with superior prognosis both before and after PSM. Advanced and metastatic disease of stage IV were often considered as unresectable, while the present study suggested that the predominant choice of treatment for thymic carcinoma should be surgical resection if feasible, even in patients with 
Table 5 Masaoka stage IV thymic carcinoma patient characteristics before and after propensity score matching

\begin{tabular}{|c|c|c|c|c|c|c|}
\hline Characteristics & \multicolumn{3}{|c|}{ Entire population } & \multicolumn{3}{|c|}{ Propensity-matched population } \\
\hline Age(years) & & & 0.298 & & & 0.362 \\
\hline$<60$ & $57(46.0)$ & $98(52.4)$ & & $23(37.1)$ & $28(45.2)$ & \\
\hline$\geq 60$ & $67(54.0)$ & $89(47.6)$ & & $39(62.9)$ & $34(54.8)$ & \\
\hline Male & $79(63.7)$ & $111(59.4)$ & & $35(56.5)$ & $40(64.5)$ & \\
\hline Female & $45(36.3)$ & $76(40.6)$ & & $27(43.5)$ & $22(35.5)$ & \\
\hline Race & & & 0.201 & & & 0.065 \\
\hline Caucasian & $94(75.8)$ & $127(67.9)$ & & $51(82.3)$ & $40(64.5)$ & \\
\hline Yes & $81(65.3)$ & $19(10.2)$ & & $19(30.6)$ & $19(30.6)$ & \\
\hline No & $43(34.7)$ & $168(89.8)$ & & $43(69.4)$ & $43(69.4)$ & \\
\hline Tumor size $(\mathrm{cm})$ & & & 0.143 & & & 0.362 \\
\hline$<7.0$ & $49(39.5)$ & $42(22.5)$ & & $24(38.7)$ & $12(19.4)$ & \\
\hline$\geq 7.0$ & $46(37.1)$ & $60(32.1)$ & & $22(35.5)$ & $17(27.4)$ & \\
\hline Unknown & $29(23.4)$ & $85(45.4)$ & & $16(25.8)$ & $33(53.2)$ & \\
\hline Distant metastasis & & & $<0.001$ & & & 0.055 \\
\hline Yes & $46(37.1)$ & $136(72.7)$ & & $30(48.4)$ & $44(71.0)$ & \\
\hline Unknown & $13(10.5)$ & $41(21.9)$ & & $9(14.5)$ & $10(16.2)$ & \\
\hline
\end{tabular}

LN, lymph node.

Masaoka stage IV. As regarding to the specific treatment, significant reductions in OS and DSS were observed in patients receiving non-total resection without radiotherapy, however, due to the limitation of sample size, we couldn't find significant difference in other treatment subgroups (total/radical resection with radiotherapy, total/radical resection without radiotherapy, and non-total resection with radiotherapy). Patients receiving total/radical resection with radiotherapy tended to yield superior survival rate, and we are looking forward to more evidence from large prospective randomized controlled trials.
An exploratory subgroup analysis was performed to identify the patients who have the best responses to surgery. Better outcome was observed in patients with younger diagnosing age, gender of female, Caucasian ethnicity, larger tumor size, with or without distant metastasis, or LN metastasis. It's the first time for a SEER study with PSM analysis to demonstrate that surgery treatment can improve survival and to determine the subgroups which benefit from the surgery intervention.

In the multivariate analysis, we found that tumor size, LN status, and distant metastasis status were prognostic 
Table 6 Univariate Kaplan-Meier analysis of OS and DSS in the matched population

\begin{tabular}{|c|c|c|c|c|c|c|}
\hline Characteristics & \multicolumn{3}{|c|}{ OS } & \multicolumn{3}{|c|}{ DSS } \\
\hline \multicolumn{7}{|l|}{ Age (years) } \\
\hline$<60$ & 43 & 22.4 & 0.727 & 42.3 & 25.2 & 0.989 \\
\hline$\geq 60$ & 49.3 & 26.3 & & 53.1 & 25.5 & \\
\hline Male & 40.2 & 11.7 & & 40.6 & 11.4 & \\
\hline Female & 55.2 & 41.7 & & 59.1 & 45.8 & \\
\hline Ethnicity & & & 0.26 & & & 0.204 \\
\hline Caucasian & 50.5 & 27.5 & & 53.5 & 28.3 & \\
\hline Yes & 58.3 & 36.1 & & 59.5 & 42.5 & \\
\hline No & 40.7 & 18.7 & & 42.1 & 16.3 & \\
\hline Surgery & & & 0.027 & & & 0.010 \\
\hline Yes & 51.9 & 32.7 & & 55.8 & 38.4 & \\
\hline No & 40.4 & 14.9 & & 40.6 & 13.1 & \\
\hline Tumor size $(\mathrm{cm})$ & & & 0.089 & & & 0.164 \\
\hline$<7.0$ & 67 & 37.1 & & 69.4 & 39.3 & \\
\hline$\geq 7.0$ & 47.4 & 26.1 & & 50.8 & 28.6 & \\
\hline Negative & 58.1 & 29 & & 59.6 & 32.4 & \\
\hline
\end{tabular}

LN, lymph node; OS, overall survival; DSS, disease-specific survival; met, metastasis.

factors on survival. These three prognostic factors were basal characteristics of patients, which represented the severity of disease, while interventional modalities such as surgery or adjuvant radiation failed to have significant impact on survival. The extent of primary tumor described as "further contiguous extension", positive LN status or with distant metastasis were classified into Masaoka stage $\mathrm{IV}$, thus, Masaoka stage IV patients included a wide range of individuals with different severity, from those who still had an opportunity for surgical intervention to those had lost surgery opportunity. In order to further discuss the impact of surgical intervention on treatable patients at Masaoka stage IV, a PSM analysis was performed to minimize the bias and make it comparable between the surgical and nonsurgical group, which revealed that surgical intervention was the only prognostic factor and independently associated with superior survival. Therefore, we demonstrated that aggressive treatment of surgery needs to be considered even in late-stage thymic carcinoma.

There have been two SEER-based studies and a nationwide database research of surgical therapy in thymic carcinoma until recently (21-23). The first 
Table 7 Cox-regression analysis for prognostic factors in the matched population

\begin{tabular}{|c|c|c|c|c|}
\hline Variables & \multicolumn{2}{|c|}{ Os } & \multicolumn{2}{|c|}{ DSS } \\
\hline \multicolumn{5}{|c|}{ Radiation treatment } \\
\hline Yes & 1 & 0.079 & 1 & 0.256 \\
\hline No & $2.051(0.924-4.554)$ & & \multicolumn{2}{|c|}{$1.425(0.773-2.624)$} \\
\hline Yes & 1 & 0.048 & 1 & 0.046 \\
\hline No & $1.985(1.007-3.913)$ & & \multicolumn{2}{|c|}{$1.649(1.009-2.697)$} \\
\hline \multicolumn{5}{|l|}{ Sex } \\
\hline Female & 1 & 0.079 & 1 & 0.122 \\
\hline Yes & 1 & 0.527 & & \\
\hline No & $1.290(0.586-2.839)$ & & & \\
\hline \multicolumn{5}{|c|}{ Tumor size (cm) } \\
\hline$<7.0$ & 1 & 0.086 & & \\
\hline$\geq 7.0$ & $1.772(0.921-3.406)$ & & & \\
\hline
\end{tabular}

$\mathrm{Cl}$, confidence interval; OS, overall survival; DSS, disease-specific survival; met, metastasis.

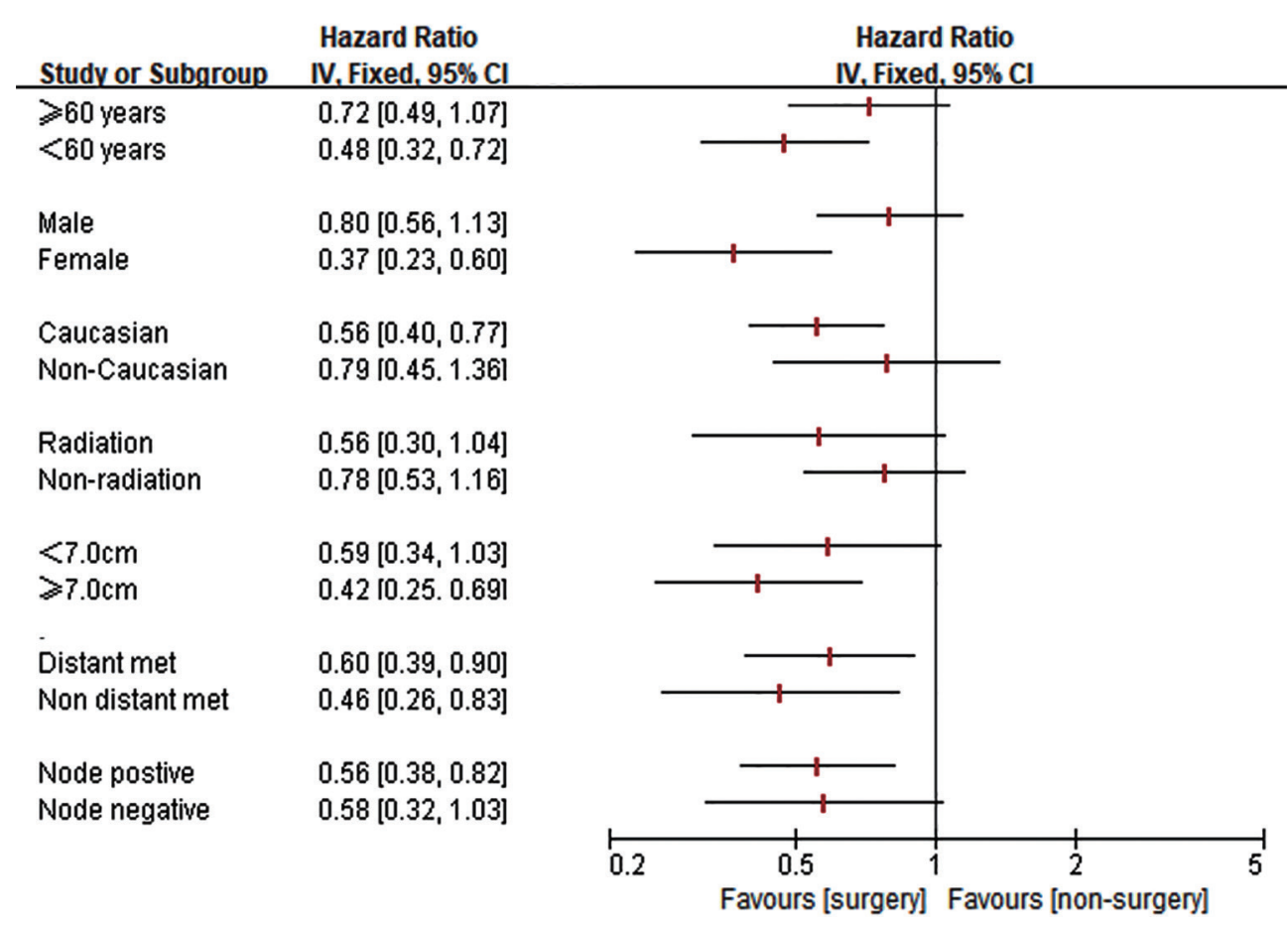

Figure 3 Result of subgroup analysis. Forest plots indicating the hazard ratio and $95 \%$ confidence interval of each subgroup. 
SEER investigation by Weksler et al. analyzed the entire thymic carcinoma population and found that type of surgical therapy was important determinants of survival [1973-2008] (22). The other study indicated that patients with debulking surgery, advanced Masaoka stage and LN metastasis had poor survival outcome (23). The nationwide database study collected from 32 Japanese institutions, evaluated the prognostic factors including clinicopathological variables and perioperative therapy for surgically treated thymic carcinoma (21). A total of 306 patients with thymic carcinoma were enrolled in this retrospective study and revealed that prognostic factors for OS were Masaoka stage and resection status, which was consistent with our result that higher pathological Masaoka stage was closely related to poor prognosis, thus we mainly focused on Masaoka stage IV tumors. However, these results of prognostic significance of surgery above were explored in the whole thymic carcinoma population, which may have bias caused by different Masaoka stages. The prognostic value of surgery therapy in late-stage thymic carcinoma should be illustrated without confounders of other stages. Therefore, we provide an initial data on the advantage of surgery treatment for late-stage thymic carcinomas based on the SEER registry.

The present analysis is different from the above previous researches in the followings: (I) the PSM analysis was performed to minimize the selection bias; (II) only thymic carcinoma of Masaoka stage IV was included; and (III) more patients were included in correspondence to the revised histology criteria. However, there are some limitations since that the SEER database didn't record the detailed surgery procedures, such as microscopical or macroscopical resection, and the clinical benefit of surgery and relapse rate are also unavailable, thus we briefly compared the surgery modality of total (total/ radical resection), non-total resection (including local tumor excision, partial removal, and debulking surgery) and no receipt of surgery, which could not fully reflect the details of surgical procedures. The Japanese nationwide study demonstrated that maximal debulking surgery might be beneficial for advanced disease deemed difficult for R0 resection, though perioperative therapy did not affect OS, it was associated with improved recurrence-free survival after R0 resection (21). Regarding to this, clinical and survival benefit of surgery and relapse rate would be worth investigating in future prospective studies.

The SEER registry used in our study is an authorized and open-assess cancer database for research purposes in the US, with a standardized reporting protocol and updated yearly. Pertinent data on demographic characteristics, tumor features, treatment modalities and follow-up data are recorded. However, some limitations still exist in this study. First is the retrospective nature of the study subjected to selection bias. Whereas the selection bias was reduced using the PSM method, we still cannot rule out some potential or unpredictable confounding factors. Further randomized controlled trials were expected to provide the prospective evidence to verify our results. Second, the information available was not comprehensive. Treatment selection criteria, patient medical history or comorbidities and recurrence status were not available in the SEER database. As a final comment, data of postoperative adjuvant therapies, such as chemotherapy and target therapy were also not provided. However, thymic carcinomas respond poorly to chemotherapy and hardly underwent target therapy, thus, this limitation could have little impact. Nonetheless, the results are striking and could affect future treatment planning.

In conclusion, our present study based on the SEER population demonstrated that administration of surgery could improve the survival outcome for patients with thymic carcinoma even at Masaoka stage IV. The selection bias of the retrospective design was reduced by PSM analysis to outline the superior therapeutic role of surgery intervention in advanced thymic carcinoma, though some potential and unpredictable confounding factors still exist due to the nature of retrospective study. Poor prognostic impact of no receipt of surgery was suggested and subgroup analysis revealed that benefit of surgery treatment was observed in patients with younger age, female sex, Caucasian ethnicity, larger tumor size, and positive LN metastasis. Further prospective explorations are highly anticipated to establish the therapeutic gain and indications of surgery in this rare malignancy.

\section{Acknowledgments}

The authors acknowledge the efforts of SEER database. Funding: None.

\section{Footnote}

Conflicts of Interest: The authors have no conflicts of interest to declare.

Ethical Statement: The authors are accountable for all aspects of the work in ensuring that questions related 
to the accuracy or integrity of any part of the work are appropriately investigated and resolved.

Open Access Statement: This is an Open Access article distributed in accordance with the Creative Commons Attribution-NonCommercial-NoDerivs 4.0 International License (CC BY-NC-ND 4.0), which permits the noncommercial replication and distribution of the article with the strict proviso that no changes or edits are made and the original work is properly cited (including links to both the formal publication through the relevant DOI and the license). See: https://creativecommons.org/licenses/by-nc-nd/4.0/.

\section{References}

1. Weissferdt A, Moran CA. Thymic carcinoma, part 1: a clinicopathologic and immunohistochemical study of 65 cases. Am J Clin Pathol 2012;138:103-14.

2. Travis WD, Brambilla E, Muller-Hermelink HK, et al. Pathology and Genetics of Tumours of the Lung, Pleura, Thymus and Heart, International Agency for Research on Cancer (IARC), Lyon, 2004.

3. Travis WD, Brambilla E, Burke AP, et al. WHO Classification of Tumours of the Lung, Pleura, Thymus and Heart, fourth ed. Lyon: International Agency for Research on Cancer (IARC), 2015.

4. Do YS, Im JG, Lee BH, et al. CT findings in malignant tumors of thymic epithelium. J Comput Assist Tomogr 1995;19:192-7.

5. Jung KJ, Lee KS, Han J, et al. Malignant thymic epithelial tumors: CT-pathologic correlation. AJR Am J Roentgenol 2001;176:433-9.

6. Tseng YL, Wang ST, Wu MH, et al. Thymic carcinoma: involvement of great vessels indicates poor prognosis. Ann Thorac Surg 2003;76:1041-5.

7. Kondo K, Monden Y. Therapy for thymic epithelial tumors: a clinical study of 1,320 patients from Japan. Ann Thorac Surg 2003;76:878-84; discussion 884-5.

8. Engels EA. Epidemiology of thymoma and associated malignancies. J Thorac Oncol 2010;5:S260-5.

9. Venuta F, Anile M, Diso D, et al. Thymoma and thymic carcinoma. Eur J Cardiothorac Surg 2010;37:13-25.

10. Lemma GL, Lee JW, Aisner SC, et al. Phase II study of carboplatin and paclitaxel in advanced thymoma and thymic carcinoma. J Clin Oncol 2011;29:2060-5.

11. Girard N. Thymic epithelial tumours: from basic principles to individualised treatment strategies. Eur
Respir Rev 2013;22:75-87.

12. National Cancer Institute. Surveillance, Epidemiology, and End Results (SEER) Program public-use data (1973-2015). Available online: http://www.seer.cancer. gov, accessed May 16, 2019.

13. Marx A, Chan JK, Coindre JM, et al. The 2015 World Health Organization Classification of Tumors of the Thymus: Continuity and Changes. J Thorac Oncol 2015;10:1383-95.

14. Wright CD. Management of thymomas. Crit Rev Oncol Hematol 2008;65:109-20.

15. Little RJ, Rubin DB. Causal effects in clinical and epidemiological studies via potential outcomes: concepts and analytical approaches. Annu Rev Public Health 2000;21:121-45.

16. Rosenbaum PR, Rubin DB. The central role of the propensity score inobservational studies for causal effects. Biometrika 1983;70:41-55.

17. Austin PC. An Introduction to Propensity Score Methods for Reducing the Effects of Confounding in Observational Studies. Multivariate Behav Res 2011;46:399-424.

18. Litvak AM, Woo K, Hayes S, et al. Clinical characteristics and outcomes for patients with thymic carcinoma: evaluation of Masaoka staging. J Thorac Oncol 2014;9:1810-5.

19. Hosaka Y, Tsuchida M, Toyabe S, et al. Masaoka stage and histologic grade predict prognosis in patients with thymic carcinoma. Ann Thorac Surg 2010;89:912-7.

20. Detterbeck FC, Nicholson AG, Kondo K, et al. The MasaokaKoga stage classification for thymic malignancies: clarification and definition of terms. J Thorac Oncol 2011;6:S1710-6.

21. Hishida T, Nomura S, Yano M, et al. Long-term outcome and prognostic factors of surgically treated thymic carcinoma: results of 306 cases from a Japanese Nationwide Database Study. Eur J Cardiothorac Surg 2016;49:835-41.

22. Weksler B, Dhupar R, Parikh V, et al. Thymic carcinoma: a multivariate analysis of factors predictive of survival in 290 patients. Ann Thorac Surg 2013;95:299-303.

23. Weksler B, Holden A, Sullivan JL. Impact of Positive Nodal Metastases in Patients with Thymic Carcinoma and Thymic Neuroendocrine Tumors. J Thorac Oncol 2015;10:1642-7.

Cite this article as: Ye C, Bao M, Li H, Liu X, Jiang G, Wang W. Surgery in Masaoka stage IV thymic carcinoma: a propensity-matched study based on the SEER database. J Thorac Dis 2020;12(3):659-671. doi: 10.21037/jtd.2019.12.111 

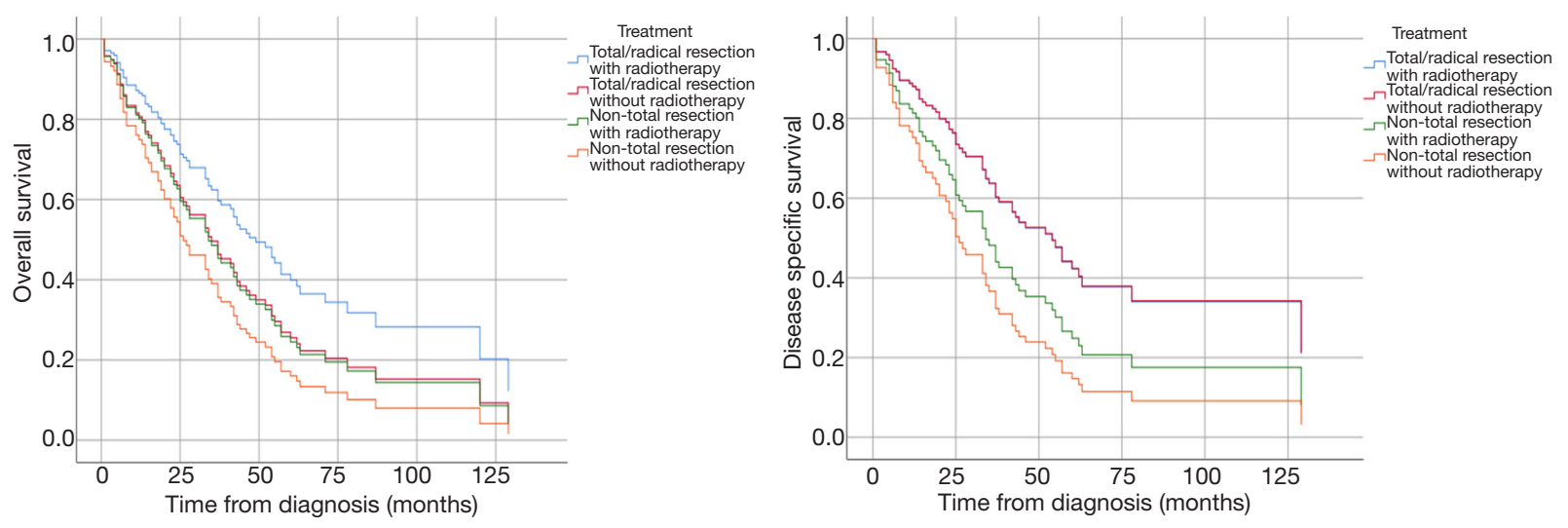

Figure S1 Survival analysis of patients with Masaoka stage IV thymic carcinoma in different treatments. (A) Overall survival analysis of patients with Masaoka stage IV thymic carcinoma in different treatments; (B) disease-specific survival analysis of patients with Masaoka stage IV thymic carcinoma in different treatments. 